Fermilab Proposal No. 362

Scientific Spokesman:

P. L. Jain

Dept. of Physics \& Astronomy State University of New York

Buffalo, New York 14214

Interaction of 200-400 GeV Pions With Emulsion Nuclei

P. L. Jain

State University of New York at Buffalo

Date Received: November 
Interaction of 200-400 GeV Pions With Emulsion Nuclei

by

Piyare L. Jain

Professor of Physics

State University of New York at Buffalo, Buffalo, N.Y.

Recently we have studied proton-Nucleon ${ }^{1}$ and proton-Nucleus ${ }^{2-4}$

interactions with 200 and $300 \mathrm{GeV}$ proton beams at FNAL. These studies have been confined to the multiplicities and to the angular distributions of the shower particles produced in proton-nucleon and proton-nucleus interactions. Low multiplicity observed in complex nuclei does not depend upon the size or on the excitation of the target nuclei and is not expressed by the internuclear cascade models. It has been found that in the target nucleus $A$, the ratio $\mathrm{R}_{\mathrm{A}}=\left\langle\mathrm{N}_{\mathrm{s}}{ }_{\mathrm{pA}} /<\mathrm{N}_{\mathrm{ch}}{ }_{\mathrm{pp}}\right.$, where $\left\langle\mathrm{N}_{\mathrm{s}}\right\rangle_{\mathrm{pp}}$ is the mean $\mathrm{p}-\mathrm{p}$ charge multiplicity and $\left\langle\mathrm{N}_{\mathrm{s}}{ }_{\mathrm{pA}}\right.$ is the mean $\mathrm{p}$-nucleus multiplicity in nucleus $A$ is practically constant and increases very slowly with $\mathrm{N}_{h}$ value. The scaling relation in emulsion is given by $\mathrm{R}_{E}=\mathrm{a}+\mathrm{bN} \mathrm{N}_{\mathrm{h}}$ where $\mathrm{a}=1$ and $b=0.06$. We also find that the rapidity distributions for p-nucleon and p-nucleus interactions are the same in the forward direction and are slightly different in the backward direction. The details of our experimental results are given in references $(1-4)$.

Now we would like to use pion as a primary particle: and compare the results of (200-400) $\mathrm{GeV}$ proton beams with the pion (200-400) GeV beams results.

\section{Exposure Details}

For each beam we would like to expose a small stack of 25 pellicles of $\mathrm{G}-5$. Illford emulsions of dimensions $10 \mathrm{~cm} \times 15 \mathrm{~cm} \times 600 \mathrm{micron}$ to a flux density of $5 \times 10^{4}$ particles $/ \mathrm{cm}^{2}$ side in such a way that the beam is parallel 
to the plane of the emulsion (along its $15 \mathrm{~cm}$ length). The pellicles shall be scanned along the tracks.

\section{References}

1. P. I. Jain, Experiments on High Energy Particles Collisions, AIP Conference Proceedings No. 12, edited by R. S. Panvini (AIP, New York 1973) p 141.

2. P. I. Jain, M. Kazuno, Z. Ahmad, B. Girard, G. Thomas, and H. Moses, Lett. Nuovo Cimento, $\underline{8}, 921$ (1973) and ㅇ, 113 (1974).

3. P. L. Jain, M. Kazuno, G. Thomas, and B. Girard, Phys. Rev. Lett. 33, 660 (1974).

4. P. L. Jain, M. Kazuno, G. Thomas, and B. Girard (Submitted for publication) 